\title{
Holographic thermodynamics of accelerating black holes
}

\author{
Andrés Anabalón, ${ }^{1}$ Michael Appels, ${ }^{2}$ Ruth Gregory, ${ }^{2,3}$ David Kubizňák, ${ }^{3}$ Robert B. Mann, ${ }^{4,3}$ and Ali Övgün ${ }^{5,6}$ \\ ${ }^{1}$ Universidad Adolfo Ibáñez, Departamento de Ciencias, Facultad de Artes Liberales, \\ Avenida Padre Hurtado 750, Viña del Mar 2520000, Chile \\ ${ }^{2}$ Centre for Particle Theory, Durham University, South Road, Durham DH1 3LE, United Kingdom \\ ${ }^{3}$ Perimeter Institute, 31 Caroline Street, Waterloo, Ontario N2L 2Y5, Canada \\ ${ }^{4}$ Department of Physics and Astronomy, University of Waterloo, Waterloo, Ontario N2L 3G1, Canada \\ ${ }^{5}$ Instituto de Física, Pontificia Universidad Católica de Valparaíso, Casilla 4950, Valparaíso, Chile \\ ${ }^{6}$ Physics Department, Arts and Sciences Faculty, Eastern Mediterranean University, \\ North Cyprus via Mersin 10, Famagusta 99628, Turkey
}

(Received 18 May 2018; revised manuscript received 23 August 2018; published 26 November 2018)

\begin{abstract}
We present a careful study of accelerating black holes in anti-de Sitter spacetime, formulating the thermodynamics and resolving discrepancies that have appeared in previous investigations of the topic. We compute the dual stress-energy tensor for the spacetime and identify the energy density associated with a static observer at infinity. The dual energy-momentum tensor can be written as a three-dimensional perfect fluid plus a nonhydrodynamic contribution with a universal coefficient which is given in gauge theory variables. We demonstrate that both the holographic computation and the method of conformal completion yield the same result for the mass. We compare to previous work on black funnels and droplets, showing that the boundary region can be endowed with noncompact geometry, and comment on this novel holographic dual geometry.
\end{abstract}

DOI: $10.1103 /$ PhysRevD.98.104038

The importance of black holes in advancing our understanding of physics cannot be underestimated. They provide a setting for testing our most fundamental ideas about gravity under extreme conditions and offer us insight into the underlying microscopic degrees of freedom that may be associated with quantum gravity. The subject of black hole thermodynamics [1-3] has proven to be an invaluable tool to this end, and broad classes of black holes have been shown to exhibit a rich and varied range of thermodynamic behavior, particularly in anti-de Sitter spacetime [4].

Within this framework, accelerating black holes have presented a unique challenge. The idealized solution is described by the $C$-metric [5-8], the spacetime of which has a stringlike singularity along one polar axis attached to the black hole. We can think of this conical singularity as a cosmic string (indeed, the conical singularity can be replaced by a finite width topological defect [9] or a magnetic flux tube [10]) with the tension providing the force driving the acceleration. Surprisingly, even though these black holes are not isolated because of the "cosmic strings," it is possible to derive sensible looking thermodynamics, although recent studies have apparently conflicting results [11-14].

Published by the American Physical Society under the terms of the Creative Commons Attribution 4.0 International license. Further distribution of this work must maintain attribution to the author(s) and the published article's title, journal citation, and DOI. Funded by SCOAP ${ }^{3}$.
We consider here the interpretation of an accelerating black hole in anti-de Sitter (AdS) spacetime, with a focus on a holographic interpretation of the thermodynamics. We resolve conflicting issues that exist in the literature, obtain a distinct set of thermodynamic variables that are now consistent with the gravitational action, and agree with both the conformal and holographic methods for computing conserved charges. To this end, we focus our attention to black holes with no acceleration horizon [15] so that there is no ambiguity as to which horizon temperature should be considered or as to whether there is an equilibrium thermodynamics for the system. In addition, as we discuss, the holographic computation and interpretation are also unambiguous and straightforward. We also comment on the cases when the acceleration horizons appear and provide a novel interpretation of the boundary geometry.

An accelerating black hole in AdS can be described by the metric $[8,15,16]$

$d s^{2}=\frac{1}{\Omega^{2}}\left[-f d t^{2}+\frac{d r^{2}}{f}+r^{2}\left(\frac{d \theta^{2}}{\Sigma}+\Sigma \sin ^{2} \theta \frac{d \phi^{2}}{K^{2}}\right)\right]$,

where

$$
\begin{aligned}
\Omega & =1+A r \cos \theta, \quad \Sigma=1+2 m A \cos \theta, \\
f(r) & =\left(1-A^{2} r^{2}\right)\left(1-\frac{2 m}{r}\right)+\frac{r^{2}}{\ell^{2}} .
\end{aligned}
$$




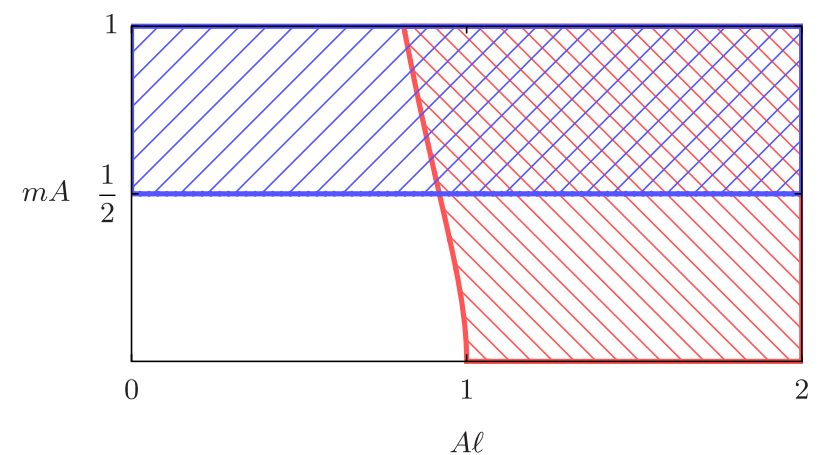

FIG. 1. Parameter space. The blue and red lines denote the boundaries in the parameter space $(m A, A \ell)$ for which the holographic computation is valid. The hashed red region is where acceleration horizons are present, and the hashed blue region is where the metric signature is not preserved, leaving the white region as the physical parameter space.

The potential $f(r)$ shows clearly the black hole nature of the solution, as well as the effects of acceleration $(A)$ and cosmological constant $\left(\Lambda=-3 / \ell^{2}\right)$. Note that we require $2 m A<1$ to preserve the metric signature. The parameter $K$ encodes information about the conical deficits on the north and south poles that have tensions given by [12]

$$
\mu_{ \pm}=\frac{\delta_{ \pm}}{8 \pi}=\frac{1}{4}\left(1-\frac{\Sigma\left(\theta_{ \pm}\right)}{K}\right)=\frac{1}{4}\left(1-\frac{1 \pm 2 m A}{K}\right) .
$$

The absence of an acceleration horizon yields the constraint $f(-1 / A \cos \theta)>0$, in turn constraining the parameter space $(m, \ell)$ to the white region bounded by the blue and red lines in Fig. 1. It is straightforward to show via a linear transformation [16] on the coordinates $(x=\cos \theta, y=-1 / A r)$ that the latter bound is equivalent to the absence of black droplets [17].

As discussed in Refs. [12,13], setting $m=0$ removes the black hole horizon and leaves pure AdS spacetime in Rindler-type coordinates. Performing the coordinate transformation [15],

$1+\frac{R^{2}}{\ell^{2}}=\frac{1+\left(1-A^{2} \ell^{2}\right) r^{2} / \ell^{2}}{\left(1-A^{2} \ell^{2}\right) \Omega^{2}}, \quad R \sin \vartheta=\frac{r \sin \theta}{\Omega}$,

recovers AdS in global coordinates,

$$
\begin{aligned}
d s_{\text {AdS }}^{2}= & -\left(1+\frac{R^{2}}{\ell^{2}}\right) \alpha^{2} d t^{2}+\frac{d R^{2}}{1+\frac{R^{2}}{\ell^{2}}} \\
& +R^{2}\left(d \vartheta^{2}+\sin ^{2} \vartheta \frac{d \phi^{2}}{K^{2}}\right),
\end{aligned}
$$

but with the notable feature that the time coordinate is not the expected AdS time being rescaled by a factor of
$\alpha=\sqrt{1-A^{2} \ell^{2}}$. Conventionally, we choose the normalization of our time coordinate so that it corresponds to the "time" of an asymptotic observer. While this is potentially a slightly slippery concept in AdS, taken together with the spherical asymptotic spatial coordinates, this scaling suggests that the correct time coordinate is not in fact $t$, but rather $\tau=\alpha t$, giving a rescaling of the time coordinate in (1). If we now proceed with this metric and compute the temperature associated with the black hole (also the temperature of the boundary field theory), then we obtain

$$
T=\frac{f^{\prime}\left(r_{+}\right)}{4 \pi \alpha}=\frac{1+3 \frac{r_{+}^{2}}{\ell^{2}}-A^{2} r_{+}^{2}\left(2+\frac{r_{+}^{2}}{\ell^{2}}-A^{2} r_{+}^{2}\right)}{4 \pi \alpha r_{+}\left(1-A^{2} r_{+}^{2}\right)},
$$

where $f\left(r_{+}\right)=0$.

It is worth pausing to reflect on this result. In past work [11-13], the standard time coordinate appearing in the AdS $\mathrm{C}$-metric was used to derive the temperature of the black hole horizon. This appeared to be a natural approach as the blackening factor of the metric was in its canonical form. However, as pointed out in Ref. [18], normalizing the time and timelike Killing vector is key to obtaining the correct thermodynamics, although the method of obtaining this correct normalization was less transparent. Here, having uncovered this suggestive result, we now proceed carefully with considering thermodynamics of the accelerating black hole. As usual, we will take the entropy to be one-quarter of the horizon area:

$$
S=\frac{\mathcal{A}}{4}=\frac{\pi r_{+}^{2}}{K\left(1-A^{2} r_{+}^{2}\right)} .
$$

The remaining task is to correctly identify the black hole mass, often the biggest challenge in studying thermodynamics of black holes with nontrivial asymptotics. In what follows, we will provide two independent arguments, beginning with the conformal completion method $[19,20]$. Although consistency of the thermodynamic relations is a common method for deriving thermodynamics (used for example in Ref. [14]), we do not consider this sufficient; hence, we return to our theme of holography, computing the holographic stress tensor of the boundary theory, thereby confirming our result. For an ancillary argument, we finally check consistency with a computation of the free energy.

The first argument uses the Ashtekar-Das definition of conformal mass [19,20], which extracts the mass via conformal regularization of the AdS C-metric near the boundary. The idea is to perform a conformal transformation on (1), $\bar{g}_{\mu \nu}=\bar{\Omega}^{2} g_{\mu \nu}$, to remove the divergence near the boundary, then obtain a conserved charge by integrating the conserved current

$$
Q(\xi)=\frac{\ell}{8 \pi} \lim _{\bar{\Omega} \rightarrow 0} \oint \frac{\ell^{2}}{\bar{\Omega}} N^{\alpha} N^{\beta} \bar{C}_{\alpha \mu \beta}^{\nu} \xi_{\nu} d \bar{S}^{\mu}
$$


composed of the Weyl tensor of the conformal metric, $\bar{C}^{\mu}{ }_{\alpha \nu \beta}$; the normal to the boundary, $N_{\mu}=\partial_{\mu} \bar{\Omega}$; and a suitable Killing vector for the mass, $\xi=\partial_{\tau}$. Even though the conformal completion is not unique, the charge thus obtained is independent of the choice of conformal completion. We pick $\bar{\Omega}=\ell \Omega r^{-1}$, which provides a smooth conformal completion in the limit $A=0$. The spacelike surface element tangent to $\bar{\Omega}=0$ is

$$
d \bar{S}_{\mu}=\delta_{\mu}^{\tau} \frac{\ell^{2}(d \cos \theta) d \phi}{\alpha K},
$$

obtained by inserting $A r \cos \theta=-1$ into the metric $\bar{g}_{\mu \nu}$ and computing the relevant determinant. This yields

$$
M=Q\left(\partial_{\tau}\right)=\alpha \frac{m}{K}
$$

for the mass, in agreement with the temperature (6), but in contrast to previous results $[11,14]$. The absence of acceleration horizons ensures that $M$ vanishes in the limit $A \ell \rightarrow 1$ only for $m=0$ and is positive otherwise.

It is now straightforward to verify the first law and Smarr [21] relation

$$
\begin{aligned}
\delta M & =T \delta S+V \delta P-\lambda_{+} \delta \mu_{+}-\lambda_{-} \delta \mu_{-}, \\
M & =2 T S-2 P V
\end{aligned}
$$

using (6), (7), and (10), provided

$$
\begin{aligned}
V & =\frac{4}{3} \frac{\pi}{K \alpha}\left[\frac{r_{+}^{3}}{\left(1-A^{2} r_{+}^{2}\right)^{2}}+m A^{2} \ell^{4}\right] \\
\lambda_{ \pm} & =\frac{1}{\alpha}\left[\frac{r_{+}}{1-A^{2} r_{+}^{2}}-m\left(1 \pm \frac{2 A \ell^{2}}{r_{+}}\right)\right]
\end{aligned}
$$

where $P=3 / 8 \pi \ell^{2}$ is the thermodynamic pressure associated with the cosmological constant [4] and $\lambda_{ \pm}$are the thermodynamic lengths introduced in Refs. $[12,13]$ that are conjugate to the tensions. We have included the possibility that the tensions vary, as otherwise the system is constrained and identification of the correct parameters can be misleading.

We now turn to another method for deriving the thermodynamic mass, by computing the holographic stress tensor. This provides an alternate and completely independent method of computation and will reveal the dual interpretation of this system. The idea here is to perform a Fefferman-Graham expansion of the metric [22], identifying the falloff of subleading terms in the metric at the boundary. These are then used to compute the dual stressenergy tensor that can be integrated to give the mass of the system.
The action, including boundary counterterms [23,24], is

$$
\begin{aligned}
I[g]= & \frac{1}{16 \pi} \int_{M} d^{4} x \sqrt{-g}\left[R+\frac{6}{\ell^{2}}\right]+\frac{1}{8 \pi} \int_{\partial M} d^{3} x \sqrt{-h} \mathcal{K} \\
& -\frac{1}{8 \pi} \int_{\partial M} d^{3} x \sqrt{-h}\left[\frac{2}{\ell}+\frac{\ell}{2} \mathcal{R}(h)\right],
\end{aligned}
$$

where $\mathcal{K}_{a b}$ is the extrinsic curvature of the boundary metric, evaluated asymptotically in an appropriate coordinate system, defined presently. $h_{a b}$ is the intrinsic metric on $\partial \mathcal{M}$, and $\mathcal{R}$ is its Ricci curvature. Varying the action gives the energy-momentum tensor:

$$
8 \pi \mathcal{T}_{a b}=\ell \mathcal{G}_{a b}(h)-\frac{2}{\ell} h_{a b}-\mathcal{K}_{a b}+h_{a b} \mathcal{K} .
$$

To compute these terms requires new coordinates near the boundary of AdS, typically parametrized by FeffermanGraham coordinates, in which

$d s^{2}=\frac{\ell^{2}}{\rho^{2}} d \rho^{2}+\frac{\rho^{2}}{\ell^{2}}\left(\gamma_{a b}^{(0)}+\frac{1}{\rho^{2}} \gamma_{a b}^{(2)}+\cdots\right) d x^{a} d x^{b}$.

Although often one identifies a $\rho$ coordinate globally, due to the complexity of (1), we instead perform an asymptotic expansion for the coordinate transformation, writing

$\frac{1}{A r}=-x-\sum F_{n}(x) \rho^{-n}, \quad \cos \theta=x+\sum G_{n}(x) \rho^{-n}$.

The functions $F_{n}$ and $G_{n}$ are fixed by the required falloff properties of (15), apart from $F_{1}$, that we choose to write as

$$
F_{1}(x)=-\frac{\left(1-A^{2} \ell^{2} X\right)^{3 / 2}}{A \omega(x) \alpha}
$$

in order to elucidate the conformal degree of freedom in the boundary metric, $\omega$, with $X=\left(1-x^{2}\right)(1+2 m A x)$. Computing this boundary metric, $d s_{(0)}^{2}=\gamma_{a b}^{(0)} d x^{a} d x^{b}$, we find it sufficient to truncate the series (16) at $n=4$ and find

$d s_{(0)}^{2}=-\omega^{2} d \tau^{2}+\frac{\omega^{2} \alpha^{2} \ell^{2} d x^{2}}{X\left(1-A^{2} \ell^{2} X\right)^{2}}+\frac{X \omega^{2} \alpha^{2} \ell^{2} d \phi^{2}}{K^{2}\left(1-A^{2} \ell^{2} X\right)}$.

Note that the transformation (16) is valid in general only when $A^{2} \ell^{2} X<1$, which is precisely the constraint that acceleration horizons are absent.

The expectation value of the energy momentum of the 3-dimensional Conformal Field Theory $\left(\mathrm{CFT}_{3}\right)$ can then be calculated, yielding 


$$
\left\langle\mathcal{T}_{a}^{b}\right\rangle=\lim _{\rho \rightarrow \infty} \frac{\rho}{\ell} \mathcal{T}_{a}^{b}=\left(\begin{array}{ccc}
-\rho_{E} & 0 & 0 \\
0 & \frac{\rho_{E}}{2}+\Pi & 0 \\
0 & 0 & \frac{\rho_{E}}{2}-\Pi
\end{array}\right),
$$

where

$$
\begin{aligned}
\rho_{E} & =\frac{m}{8 \pi \ell^{2} \alpha^{3} \omega^{3}}\left(1-A^{2} \ell^{2} X\right)^{3 / 2}\left(2-3 A^{2} \ell^{2} X\right), \\
\Pi & =\frac{3 m A^{2} X}{16 \pi \alpha^{3} \omega^{3}}\left(1-A^{2} \ell^{2} X\right)^{\frac{3}{2}} .
\end{aligned}
$$

We can reexpress this in the language of the fluid/gravity correspondence (for a review and references, see Ref. [25]) as

$$
\left\langle\mathcal{T}_{a b}\right\rangle=\frac{3}{2} d(x) U_{a} U_{b}+\frac{d(x)}{2} \gamma_{a b}^{(0)}+\xi \Theta_{a b},
$$

where the 4-velocity $U$ and $\Theta$ are defined as

$$
\begin{aligned}
U & =\sqrt{\frac{3-2 A^{2} \ell^{2} X}{3 \omega\left(1-A^{2} \ell^{2} X\right)}} \partial_{\tau}-\frac{A X}{\sqrt{3 \omega} \alpha} \sqrt{\left(1-A^{2} \ell^{2} X\right)} \partial_{x}, \\
\Theta_{a b} & =C_{a b d} U^{d}+C_{b a d} U^{d},
\end{aligned}
$$

with $C_{a b c}=\nabla_{[c} R_{b] a}-g_{a[b} \nabla_{c]} R / 4$ the Cotton tensor [26] of the metric (18); boundary indices are raised and lowered with $\gamma_{a b}^{(0)}$.

The fact that the boundary is nonconformally flat leads to the inclusion of nonhydrodynamic corrections to the perfect fluid, with

$$
d(x)=\frac{m \sqrt{\left(1-A^{2} \ell^{2} X\right)^{5}}}{4 \pi \ell^{2} \omega^{3} \alpha^{3}} .
$$

The universal (in the sense of independent of black hole mass and acceleration) coefficient is

$$
\xi=\frac{\ell^{2}}{8 \pi \sqrt{3}}=\sqrt{\frac{2}{3}} \frac{1}{12 \pi} k^{1 / 2} N^{3 / 2}
$$

using the standard $\mathrm{AdS}_{4} / \mathrm{CFT}_{3}$ dictionary to identify $k$ with the level and $N$ with the rank of the gauge groups of the $N=6$ Super Chern-Simons theory. All dissipative corrections enumerated in Ref. [25] are seen to vanish, ensuring the uniqueness of the decomposition given in (21).

Integrating the energy density, measured with respect to a static geodesic observer $\rho_{E}$, yields

$$
M=\int \rho_{E} \sqrt{-\gamma^{(0)}} d x d \phi=\frac{\alpha m}{K},
$$

for the mass, in agreement with (10) and independent of the conformal frame (the choice of $\omega$ ).

We see from (18) that the boundary metric does not satisfy Dirichlet boundary conditions. However, for arbitrary variations of the parameters $A$ and $m$, we find that

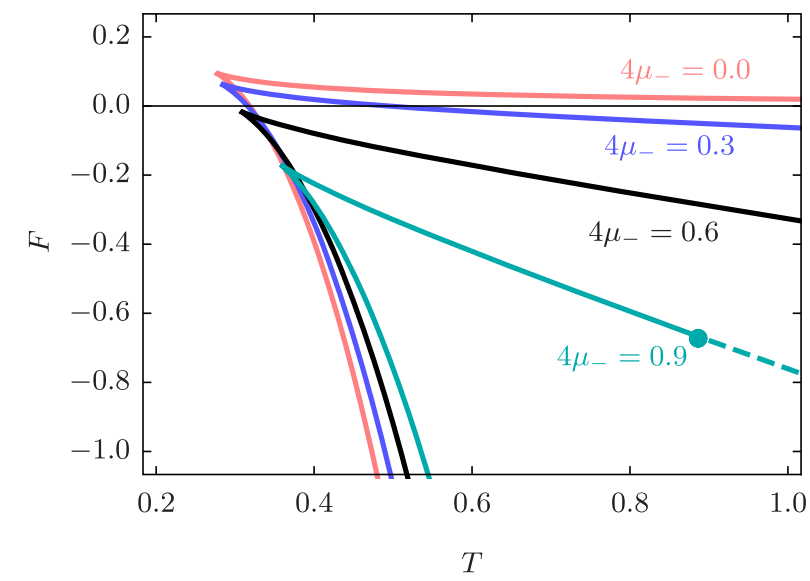

FIG. 2. Free energy. The red curve is the Schwarzschild-AdS case, illustrating the well-known Hawking-Page transition, situated at a temperature given by the intersection of the red curve with $F=0$. We do not know of any such interpretation for all other curves with $\mu \neq 0$. The upper parts of these curves do not continue to arbitrarily large $M$ but terminate at the boundary given in Fig. 1; this is visible in the above plot only for $4 \mu_{-}=0.9$.

$\delta I[g]=0$, provided we set $\alpha=\sqrt{1-A^{2} \ell^{2}}$. Our analysis therefore points toward the possibility of generalizing the conditions under which the conformal and holographic methods coincide for the mass computation [27,28].

Finally, let us return to the computation of the action (26). We find

$$
I=\frac{\beta}{2 \alpha K}\left(m-2 m A^{2} \ell^{2}-\frac{r_{+}^{3}}{\ell^{2}\left(1-A^{2} r_{+}^{2}\right)^{2}}\right),
$$

using the time coordinate $\tau$. Some simple algebra then yields the expected result $F=I / \beta=M-T S$ for the free energy, which we plot (for fixed pressure) in Fig. 2.

Although similar in form, the behavior of the free energy no longer indicates the presence of a standard HawkingPage transition [29]. As the string tension is fixed for the curves in the plot, no transition to pure radiation (with zero tension) is possible. One may, however, speculate that a transition to a different type of spacetime (for example that of the expanding spherical wave with an attached semiinfinite string of given tension, similar to that in Ref. [30]) may still be possible-such an investigation, however, remains to be carried out.

We can also explore the isoperimetric ratio, or the ratio of the volume to the areal radius: $\mathcal{R}=\left(\frac{3 V}{\omega_{2}}\right) \frac{1}{3}\left(\frac{\omega_{2}}{\mathcal{A}}\right)^{\frac{1}{2}}$ (recall $\omega_{2}=$ $4 \pi / K$ here). Using (7) and (12), we find $\mathcal{R} \geq 1$, indicating it satisfies the standard reverse isoperimetric inequality [31], not adding to the notable exceptions [32-34].

Our full and consistent description of the thermodynamics of an accelerating black hole reconciles discrepancies and conflicts that have appeared in previous investigations of this system $[11,12,14]$. For example, while a set of thermodynamic variables for charged accelerating black 
holes respecting the first law was obtained [11-13], the computations employed an incorrectly normalized Killing vector at infinity; furthermore, the resultant free energy is not consistent with the standard Euclidean action calculation. Alternate expressions for mass and temperature have been posited [14], with the tension of one deficit held fixed to zero. The other tension, while allowed to vary, was not included in the first law, which was derived by assuming integrability of a scaling of mass and temperature. However no physical interpretation was given either for this scaling or for why the energy content of the tension was thermodynamically irrelevant. Furthermore, the vacuum accelerating black hole has an acceleration horizon, akin to a Rindler horizon, and the full structure of the spacetime is that of two accelerating black holes in two Rindler regions. Whether one should be considering a single thermodynamic mass and first law with an additional horizon and black hole or whether, as suggested in Ref. [35], this should be considered as a single system with a mass dipole is an open question.

We also found a decomposition of the dual stress-energy tensor for the accelerating black hole in terms of a perfect fluid plus conformal tensors. We obtained a new "universal" coefficient, $\xi$, that is relevant for the fluid/gravity correspondence in nonconformally flat manifolds. It is natural to expect the existence of nonhydrodynamic corrections to the energy-momentum tensor for an evendimensional CFT due to the conformal anomalies. We have shown here that a similar picture arises in the odddimensional case by explicitly constructing the relevant nonhydrodynamic corrections necessary to provide a complete holographic description of the system, cf. Ref. [36].

It would also be interesting to make a connection with the weak coupling calculation of stress tensors in the presence of conical deficits [37]. Future work will involve investigating accelerating black holes with rotation, scalar fields [38,39], and charge. The latter system will be a challenge due to the asymptotic structure of the gauge field.

Since our computation is independent of the conformal frame, we can compare to investigations of holographic $\mathrm{C}$-metrics with an acceleration horizon. For example, by choosing $\omega^{2}=\left(1-A^{2} \ell^{2} X\right) \alpha^{-2}$, we recover the form of the boundary metric employed in Ref. [17], and our coordinate transformation (16) is now valid throughout $x \in[-1,1]$. However, if the condition $A^{2} \ell^{2} X<1$ is violated, then a black droplet/black funnel is present, and we no longer have an equilibrium temperature for the system in general. The boundary geometry corresponds to a black hole in a spatially compact universe, and so there is no spatial asymptotic region as pointed out in Ref. [17]. However, with the full conformal degree of freedom present in our expression, we can easily remedy this shortcoming by, for example, multiplying the $\omega$ above by $\frac{1}{\sqrt{1-x}}$, giving an $\operatorname{AdS}_{2} \times S^{1}$ asymptotic region at $x=1$ with the $\mathrm{AdS}_{2}$ and $S^{1}$ radii being equal. If we again multiply by $\frac{1}{\sqrt{1-x^{2}}}$, then there are actually two $\mathrm{AdS}_{2} \times S^{1}$ asymptotic regions at $x= \pm 1$, and $\gamma_{a b}^{(0)}$ yields the geometry of a wormhole when there are no horizons at the boundary. The $\mathrm{AdS}_{2} \times S^{1}$ asymptotic geometry is supersymmetric and to our knowledge has been unnoticed so far in the literature.

\section{ACKNOWLEDGMENTS}

We thank Alex Buchel, Rob Myers, Jiř́ Podolský, Mukund Rangamani, and Kostas Skenderis for valuable discussions. This work was supported in part by the Chilean FONDECYT [Grants No. 1181047 (A. A.), No. 1170279 (A. A.), No. 1161418 (A. A.), and No. 3170035 (A. Ö.)], CONICYT-RCUK [Newton-Picarte Grants No. DPI20140053 (A. A.) and No. DPI20140115 (A. A.)], by the STFC [consolidated Grant No. ST/P000371/ 1 (R. G.)], by the Perimeter Institute for Theoretical Physics (R. G. and D. K.), and by the Natural Sciences and Engineering Research Council of Canada (D. K. and R. M.). M. A. and A. O. would like to thank Perimeter Institute and University of Waterloo for hospitality. Research at Perimeter Institute is supported by the Government of Canada through the Department of Innovation, Science and Economic Development Canada and by the Province of Ontario through the Ministry of Research, Innovation and Science.
[1] J. D. Bekenstein, Black holes and entropy, Phys. Rev. D 7, 2333 (1973).

[2] J. D. Bekenstein, Generalized second law of thermodynamics in black hole physics, Phys. Rev. D 9, 3292 (1974).

[3] S. W. Hawking, Particle creation by black holes, Commun. Math. Phys. 43, 199 (1975); Erratum, Commun. Math. Phys. 46, 206(E) (1976).
[4] D. Kubizňák, R. B. Mann, and M. Teo, Black hole chemistry: Thermodynamics with Lambda, Classical Quantum Gravity 34, 063001 (2017).

[5] W. Kinnersley and M. Walker, Uniformly accelerating charged mass in general relativity, Phys. Rev. D 2, 1359 (1970).

[6] J. F. Plebanski and M. Demianski, Rotating, charged, and uniformly accelerating mass in general relativity, Ann. Phys. (N.Y.) 98, 98 (1976). 
[7] O. J. C. Dias and J. P. S. Lemos, Pair of accelerated black holes in anti-de Sitter background: AdS C metric, Phys. Rev. D 67, 064001 (2003).

[8] J. B. Griffiths and J. Podolsky, A New look at the PlebanskiDemianski family of solutions, Int. J. Mod. Phys. D 15, 335 (2006).

[9] R. Gregory and M. Hindmarsh, Smooth metrics for snapping strings, Phys. Rev. D 52, 5598 (1995).

[10] F. Dowker, J. P. Gauntlett, D. A. Kastor, and J. H. Traschen, Pair creation of dilaton black holes, Phys. Rev. D 49, 2909 (1994).

[11] M. Appels, R. Gregory, and D. Kubizňák, Thermodynamics of Accelerating Black Holes, Phys. Rev. Lett. 117, 131303 (2016).

[12] M. Appels, R. Gregory, and D. Kubizňák, Black hole thermodynamics with conical defects, J. High Energy Phys. 05 (2017) 116.

[13] R. Gregory, Accelerating black holes, J. Phys. Conf. Ser. 942, 012002 (2017).

[14] M. Astorino, Thermodynamics of regular accelerating black holes, Phys. Rev. D 95, 064007 (2017).

[15] J. Podolsky, Accelerating black holes in anti-de Sitter universe, Czech. J. Phys. 52, 1 (2002).

[16] K. Hong and E. Teo, A new form of the C metric, Classical Quantum Gravity 20, 3269 (2003).

[17] V. E. Hubeny, D. Marolf, and M. Rangamani, Black funnels and droplets from the AdS C-metrics, Classical Quantum Gravity 27, 025001 (2010).

[18] G. W. Gibbons, M. J. Perry, and C. N. Pope, The first law of thermodynamics for Kerr-anti-de Sitter black holes, Classical Quantum Gravity 22, 1503 (2005).

[19] A. Ashtekar and S. Das, Asymptotically anti-de Sitter spacetimes: Conserved quantities, Classical Quantum Gravity 17, L17 (2000).

[20] S. Das and R. Mann, Conserved quantities in Kerr-anti-de Sitter space-times in various dimensions, J. High Energy Phys. 08 (2000) 033.

[21] L. Smarr, Mass Formula for Kerr Black Holes, Phys. Rev. Lett. 30, 71 (1973); Erratum, Phys. Rev. Lett. 30, 521(E) (1973).

[22] C. Fefferman and C. Robin Graham, Conformal invariants in Elie Cartan et les Mathématiques d'aujourd 'hui, Numero Hors Serie (Astérisque, Lyon, 1985), p. 95.

[23] V. Balasubramanian and P. Kraus, A stress tensor for anti-de Sitter gravity, Commun. Math. Phys. 208, 413 (1999).
[24] R. B. Mann, Misner string entropy, Phys. Rev. D 60, 104047 (1999).

[25] M. Rangamani, Gravity and hydrodynamics: Lectures on the fluid-gravity correspondence, Classical Quantum Gravity 26, 224003 (2009).

[26] A. Mukhopadhyay, A. C. Petkou, P. M. Petropoulos, V. Pozzoli, and K. Siampos, Holographic perfect fluidity, cotton energy-momentum duality and transport properties, J. High Energy Phys. 04 (2014) 136.

[27] S. Hollands, A. Ishibashi, and D. Marolf, Comparison between various notions of conserved charges in asymptotically AdS-spacetimes, Classical Quantum Gravity 22, 2881 (2005).

[28] I. Papadimitriou and K. Skenderis, Thermodynamics of asymptotically locally AdS spacetimes, J. High Energy Phys. 08 (2005) 004.

[29] S. W. Hawking and D. N. Page, Thermodynamics of black holes in anti-de Sitter space, Commun. Math. Phys. 87, 577 (1983).

[30] J. Podolsky and J. B. Griffiths, A Snapping cosmic string in a de Sitter or anti-de Sitter universe, Classical Quantum Gravity 21, 2537 (2004).

[31] M. Cvetic, G. W. Gibbons, D. Kubizňák, and C. N. Pope, Black hole enthalpy and an entropy inequality for the thermodynamic volume, Phys. Rev. D 84, 024037 (2011).

[32] R. A. Hennigar, D. Kubizňák, and R. B. Mann, Entropy Inequality Violations from Ultraspinning Black Holes, Phys. Rev. Lett. 115, 031101 (2015).

[33] R. A. Hennigar, D. Kubizňák, R. B. Mann, and N. Musoke, Ultraspinning limits and super-entropic black holes, J. High Energy Phys. 06 (2015) 096.

[34] W. G. Brenna, R. B. Mann, and M. Park, Mass and thermodynamic volume in lifshitz spacetimes, Phys. Rev. D 92, 044015 (2015).

[35] K. Dutta, S. Ray, and J. Traschen, Boost mass and the mechanics of accelerated black holes, Classical Quantum Gravity 23, 335 (2006).

[36] G. Bernardi de Freitas and H. S. Reall, Algebraically special solutions in AdS/CFT, J. High Energy Phys. 06 (2014) 148.

[37] J. S. Dowker, Quantum field theory on a cone, J. Phys. A 10, 115 (1977).

[38] A. Anabalon and H. Maeda, New charged black holes with conformal scalar hair, Phys. Rev. D 81, 041501 (2010).

[39] A. Anabalon, Exact black holes and universality in the backreaction of non-linear Sigma Models with a potential in (A)dS4, J. High Energy Phys. 06 (2012) 127. 